\title{
Dapagliflozin Therapy Curative Effect Observation on Nonalcoholic Fatty Liver Disease in Patients with Type 2 Diabetes Mellitus
}

\author{
CHEN LIANG. HU*, YU CHUAN. WANG, YU. XI AND XIN MING. YAO1
}

Department of endocrinology, People's Hospital of Huangshan, Huangshan, Anhui 245000, China; ${ }^{1}$ Department of Endocrinology, Yijishan Hospital of Wannan Medical College, Wuhu, Anhui 241000, China

Hu et al.: Dapagliflozin therapy curative effect observation on nonalcoholic fatty liver disease

To explore whether dagglitazine treatment is beneficial to the improvement of nonalcoholic fatty liver in patients with type 2 diabetes. We selected sixty patients with type 2 diabetes and nonalcoholic fatty liver disease from April 2019 to December 2019 in our hospital. If the patients had been with Glycosylated hemoglobin $\geq 9 \%$ or fasting blood glucose $\geq 11.1 \mathrm{mmol} / \mathrm{L}$, they were given continuous subcutaneous insulin infusion treatment to improve high glucose toxicity. After that, these patients were randomly divided into two groups: one group of patients was treated with dapagliflozin; the other group was treated with Metformin. If the patients had been with Glycosylated hemoglobin $<9 \%$ or fasting blood glucose $<11.1$ $\mathrm{mmol} / \mathrm{L}$, they were directly randomly divided to dapagliflozin treatment group or Metformin treatment group. Our random division made sure that there were 30 patients in each group. After discharge, the patients maintained the original treatment plan for $12 \mathrm{w}$. The main observational indexes were observed, measured and compared before and after treatment. The body weight, body mass index, waist circumference, waist to hip ratio, systolic pressure, diastolic pressure, total cholesterol, triglyceride, lowdensity lipoprotein cholesterol, high-density lipoprotein cholesterol, urinary albumin to creatinine ratio, blood uric acid, fasting blood glucose, fasting insulin, glycosylated hemoglobin, insulin resistance index, $\beta$ cell function index, $\Delta \mathrm{I30} / \Delta \mathbf{G 3 0}$, alanine aminotransferase, measure controlled attenuation parameter and liver stiffness measurement were treated. $T$ test was used to comparison of baseline level of each index before treatment between the two groups, and difference of each index before and after treatment for two groups. There was no difference between the two groups before treatment. After 12 w of metformin treatment, fasting blood glucose and glycosylated hemoglobin in the control group decreased significantly, compared with those before treatment $(\mathbf{p}<0.05)$. Fasting insulin, $\beta$ cell function index and $\Delta \mathrm{I30} / \Delta \mathrm{G30}$ in the control group decreased significant, compared with those before treatment $(p<0.05)$. There was no significant difference in body weight, body mass index, waist circumference, waist to hip ratio, systolic and diastolic blood pressure, total cholesterol, triglycerides, high-density lipoprotein cholesterol, low-density lipoprotein cholesterol, urinary albumin-creatinine ratio, blood uric acid, insulin resistance index, alanine aminotransferase, liver stiffness measurement and controlled attenuation parameters after and before treatment in the control group $(\mathrm{p}>\mathbf{0 . 0 5})$. After $12 \mathrm{w}$ of treatment, body mass index, waist circumference, waist hip ratio, systolic blood pressure, diastolic blood pressure, triglycerides, fasting blood glucose, glycosylated hemoglobin and insulin resistance index of dagglitazine group decreased significantly compared with those before treatment $(\mathbf{p}<\mathbf{0 . 0 5})$. While fasting insulin, $\beta$ cell function index, $\Delta \mathbf{I 3 0} / \Delta \mathbf{G 3 0}$ increased significantly compared with those before treatment $(p<0.05)$. Alanine aminotransferase, liver stiffness measurement and controlled attenuation parameters decreased significantly compared with those before treatment $(\mathbf{p}<\mathbf{0 . 0 5})$. Dagglitazine is beneficial to the improvement of nonalcoholic fatty liver in patients with type 2 diabetes mellitus. The mechanism may be related to the decrease of body mass index, waist circumference, waist to hip ratio, triglycerides and insulin resistance.

Key words: Dagglitazine; Type 2 diabetes mellitus; nonalcoholic fatty liver; transient elastography

In developed countries, nonalcoholic fatty liver disease (NAFLD) has become one of the common chronic diseases, with an incidence rate of $20 \%$ to $40 \%{ }^{[1]}$. The incidence of non-alcoholic fatty liver in China in the

*Address for correspondence

E-mail: huchenliang1976122@126.com

Special Issue 7, 2020 
past $10 \mathrm{y}$ has also shown a significant upward trend. A retrospective study on the incidence of non-alcoholic fatty liver in China had indicated that the incidence of non-alcoholic fatty liver in economically developed areas such as Beijing and Shanghai increased from $15 \%$ to $30 \%{ }^{[2]}$. Kwok R, et al. ${ }^{[3]}$ study had shown that obesity, abnormal lipid metabolism and elevated blood sugar are high-risk factors for the incidence of nonalcoholic fatty liver, and the incidence of non-alcoholic fatty liver in diabetic patients was as high as $70 \%$. Diabetes and non-alcoholic fatty liver often coexist in the same patient ${ }^{[4]}$, which can increase the incidence of chronic vascular complications in patients ${ }^{[5]}$. At the same time, diabetes may increase the occurrence of liver cirrhosis and hepatocellular carcinoma in patients with non-alcoholic fatty liver ${ }^{[6]}$. Dapagliflozin is a new type of hypoglycemic drug. It is a sodiumglucose cotransporter 2 (SGLT-2) inhibitor. It inhibits the SGLT-2 protein, reduces the reabsorption of glucose in the kidney, and increases the excretion of blood glucose from the urine. There by reducing blood sugar. Some studies have found that dapagliflozin can reduce the occurrence of complications of non-alcoholic steatohepatitis in rodent models $s^{[7,8]}$. There are few studies on whether dapagliflozin treatment is beneficial to the improvement of non-alcoholic fatty liver in diabetic patients. In this study, we explored the related liver enzyme indexes after dapagliflozin and metformin treated two groups of patients with type 2 diabetes and non-alcoholic fatty liver, and transient elastography was used to measure liver controlled attenuation parameters (CAP) and liver stiffness (LSM) The difference in changes in other indicators is to understand whether dapagliflozin treatment is beneficial to the improvement of non-alcoholic fatty liver in diabetic patients.

\section{MATERIAL AND METHODS}

\section{Research object:}

60 columns of patients with diabetes and non-alcoholic fatty liver were hospitalized in our hospital from April 2019 to December 2019.

Inclusion criteria: All met the 1999 World Health Organization (WHO) diagnostic criteria for type 2 diabetes; Diabetes duration $\leq 2$ y; Age 20-65 y; Color Doppler ultrasound diagnosis of fatty liver.

Exclusion criteria: Type 1 diabetes; Diabetes acute and chronic complications; Severe infection; Severe liver and kidney insufficiency, cardiac insufficiency; Longterm heavy drinkers (drinking equivalent The amount of ethanol is $\geq 140 \mathrm{~g} / \mathrm{w}$ for men and $\geq 70 \mathrm{~g} / \mathrm{w}$ for women); have autoimmune liver disease, viral hepatitis, druginduced liver disease, and cirrhosis.

All patients signed an informed consent form, and the study was reviewed by the Medical Ethics Committee of Huangshan People's Hospital.

\section{Data Collection:}

Measure the height, weight, waist circumference, hip circumference and blood pressure of the patient after admission, and take fasting venous blood the next morning to check fasting blood glucose (FPG), insulin, $\mathrm{C}$ peptide, liver function, kidney function, blood lipids, and blood uric acid, Glycosylated hemoglobin (HbA1C) and urinary albumin-creatinine ratio (ACR), followed by oral glucose tolerance test (OGTT) (100 $\mathrm{g}$ of steamed bread meal) and check blood glucose and plasma insulin and $\mathrm{C}$ peptide levels for $0.5,1,2$ and $3 \mathrm{~h}$. Hepatobiliary, pancreatic and spleen color doppler ultrasound examination and liver transient elastography examination were performed on an empty stomach during hospitalization to determine LSM and CAP. The LSM and CAP measuring instruments is the FT7000 shear wave tissue quantitative ultrasonic diagnostic instrument produced by Wuxi Hisky Medical Technology Co., Ltd.

\section{Treatment and grouping:}

All patients are given diet and lifestyle guidance. When patients with $\mathrm{HbA} 1 \mathrm{C} \geq 9 \%$ or $\mathrm{FPG} \geq 11.1 \mathrm{mmol} / \mathrm{L}$ were given continuous subcutaneous insulin infusion (CSII) intensive treatment to improve high glucose toxicity, they were randomly divided into two groups, one group was treated with dapagliflozin $50 \mathrm{mg}$ qd hypoglycemic therapy, and the other One group was treated with metformin 0.5 tid hypoglycemic treatment; when patients with $\mathrm{HbA} 1 \mathrm{C}<9 \%$ or $\mathrm{FPG}<11.1$ $\mathrm{mmol} / \mathrm{L}$, one group was treated with dapagliflozin 50mg qd hypoglycemic treatment, and the other group was treated with metformin 0.5 tid Hypoglycemic treatment; if patients with hypertension continue to maintain the original antihypertensive treatment or adjust the antihypertensive program appropriately, they will be discharged after the blood sugar stabilizes. After discharge from the hospital, each group continued the hypoglycemic treatment before discharge, and self-checked capillary blood glucose with a portable blood glucose meter at home. After half a mo from the hospital, he went to our clinic for the first follow-up, and continued the original treatment without special 
side effects. After the 12-w course of total medication was completed, I went to our clinic for follow-up. The diabetes specialist nurse measured the patient's height, weight, waist circumference, hip circumference and blood pressure, and took fasting venous blood to check blood sugar, insulin, $\mathrm{C}$ peptide, liver function, kidney function, Blood lipids, blood uric acid, HbA1C and urinary albumin- ACR, blood glucose, insulin, and C-peptide were checked for $0.5 \mathrm{~h}$ after a meal of 100 $\mathrm{g}$ of steamed buns, and transient elastography of the liver was performed to determine LSM and CAP during fasting.

\section{Observation indicators:}

Calculate body mass index (BMI) $=$ weight $/$ height $^{2}$. Waist-to-hip ratio (WHR) =waist circumference/ hip circumference, HOME- $\beta=20 \times$ FINS/(FPG-3.5), HOMA-IR $=$ FBG $\cdot$ FINS/22.5, $\quad \Delta^{\mathrm{I} 30} / \Delta^{\mathrm{G} 30}=(\mathrm{I} 30-\mathrm{I} 0) /$ (G30-G0). Observe the weight (Weight), BMI, waist circumference (Waist), waist-to-hip ratio (WHR), systolic blood pressure (SBP), diastolic blood pressure (DBP), cholesterol (TC), triglycerides (TG) of each group before and after treatment, High-density lipoprotein cholesterol (HDL), low-density lipoprotein cholesterol (LDL), blood uric acid (BUA), urine albumin-creatinine ratio (ACR), fasting blood glucose (FBG), fasting insulin (FINS), HbA1C, HOMEChanges in general indicators such as $\beta$, HOMA-IR, $\Delta^{\mathrm{I} 30} / \Delta^{\mathrm{G} 30}$, and liver-related indicators such as alanine aminotransferase (ALT), LSM and CAP.

\section{Statistical analysis:}

The SAS 9.2 software was used for analysis, and each index was expressed as $\mathrm{x} \pm \mathrm{s}$. The difference between groups before treatment was compared by two independent sample t-tests, and the comparison of indicators within the group before and after treatment was performed by paired t-test. Two independent samples $\mathrm{t}$ test was used. $\mathrm{p}<0.05$ was considered statistically significant.

\section{RESULT AND DISCUSSION}

Sixty patients with diabetes and fatty liver who were hospitalized in our department were selected with no loss to follow-up. There were 30 cases in the dapagliflozin group, including 6 females and 24 males; and 30 cases in the dapagliflozin group (control group), including 7 females, 23 males. All patients completed $12 \mathrm{w}$ of treatment. One patient in the dapagliflozin group had urine leukocytosis at the first follow-up, and no other symptoms. He was asked to pay attention to personal hygiene and drink plenty of water. After half a mo, the urine routine was normal again. In the metformin group, two cases of gastrointestinal adverse reactions occurred, which were changed to metformin for 0.25 tid and improved after treatment. After half a mo, the dose was increased to 0.5 tid again, and there was no intolerance until the end of treatment. There were no symptoms of hypoglycemia and monitored hypoglycemia (blood sugar $<3.8 \mathrm{mmol} / \mathrm{L}$ ) in both groups.

The age, weight, BMI, waist circumference, waistto-hip ratio, systolic blood pressure, diastolic blood pressure, TC, TG, HDL, LDL, ACR, blood uric acid, fasting blood glucose, fasting insulin, HOME- $\beta$, HOMA-IR, $\Delta^{\mathrm{I} 30} / \Delta^{\mathrm{G} 30}, \mathrm{HbA} 1 \mathrm{C}, \mathrm{ALT}, \mathrm{LSM}$ and CAP were not statistically significant $(p>0.05)$, and they were comparable, as shown in TABLE 1.

The fasting blood glucose and $\mathrm{HbA} 1 \mathrm{C}$ after treatment in the control group were lower than before treatment,

\begin{tabular}{|c|c|c|}
\hline $\begin{array}{l}\text { TABLE 1: } \\
\text { IMFORMATIO }\end{array}$ & $\begin{array}{l}\text { COMPARISON } \\
\text { BETWEEN TW }\end{array}$ & $\begin{array}{c}\text { OF GENERA } \\
\text { SROUPS ( } \bar{x} \pm s)\end{array}$ \\
\hline Item & Control $(n=30)$ & Treatment $(n=30)$ \\
\hline Sex $(M / F)$ & $23 / 7$ & $24 / 6$ \\
\hline Age (year) & $52.1 \pm 10.2$ & $48.9 \pm 10.6$ \\
\hline Weight (kg) & $76.45 \pm 9.17$ & $78.51 \pm 12.6$ \\
\hline BMI $\left(\mathrm{kg} / \mathrm{m}^{2}\right)$ & $25.82 \pm 6.67$ & $27.71 \pm 3.29$ \\
\hline Waist $(\mathrm{cm})$ & $97.03 \pm 7.17$ & $98.43 \pm 9.76$ \\
\hline WHR & $0.96 \pm 0.06$ & $0.97 \pm 0.07$ \\
\hline SBP (mmHg) & $133.86 \pm 19.09$ & $133.06 \pm 17.74$ \\
\hline $\mathrm{DBP}(\mathrm{mmHg})$ & $88.6 \pm 9.59$ & $90.13 \pm 11.40$ \\
\hline TC (mmol/L) & $4.97 \pm 1.21$ & $4.62 \pm 1.07$ \\
\hline TG (mmol/L) & $2.52 \pm 0.87$ & $3.72 \pm 3.23$ \\
\hline $\mathrm{HDL}(\mathrm{mmol} / \mathrm{L})$ & $3.30 \pm 1.11$ & $1.95 \pm 5.23$ \\
\hline LDL (mmol/L) & $3.31 \pm 2.02$ & $2.98 \pm 0.69$ \\
\hline ACR $(\mathrm{mg} / \mathrm{g})$ & $18.04 \pm 31.06$ & $14.95 \pm 19.83$ \\
\hline BUA (umol/L) & $356.90 \pm 78.46$ & $334.1 \pm 78.46$ \\
\hline FBG (mmol/L) & $11.24 \pm 3.57$ & $13.05 \pm 3.68$ \\
\hline Fins (uU/L) & $6.82 \pm 4.45$ & $7.88 \pm 4.59$ \\
\hline HOME-B & $19.63 \pm 16.13$ & $19.50 \pm 13.50$ \\
\hline HOME-IR & $3.98 \pm 2.21$ & $4.41 \pm 2.50$ \\
\hline $\begin{array}{l}\Delta^{130} / \Delta^{\mathrm{G} 30}(\mathrm{uU} / \\
\mathrm{mmol})\end{array}$ & $1.03 \pm 1.63$ & $1.73 \pm 2.40$ \\
\hline HbA1C (\%) & $11.2 \pm 2.29$ & $11.45 \pm 2.09$ \\
\hline ALT (U/L) & $38.14 \pm 39.67$ & $36.5 \pm 19.80$ \\
\hline LSM (KPa) & $6.65 \pm 2.74$ & $7.74 \pm 2.83$ \\
\hline $\mathrm{CAP}(\mathrm{dB} / \mathrm{m})$ & $298 \pm 24.03$ & $300.63 \pm 24.83$ \\
\hline
\end{tabular}

Control: Treatment with Metformin; Treatment: treatment with dapagliflozin; BMI: Body mass index; WHR: Waist hip ratio; SBP: Systolic blood pressure; DBP: Diastolic blood pressure; TC: Total cholesterol; TG: Triglyceride; HDL-C: High-density lipoprotein cholesterol; LDL-C: Low-density lipoprotein cholesterol; ACR: Urinary albumin to creatinine ratio; BUA: Blood uric acid; FBG: Fasting plasma glucose; Fins: Fasting insulin; HOMA-B: B cell function index; HOMA-IR: Insulin resistance index; HbA1c: Glycosylated hemoglobin; ALT: Alanine aminotransferase; CAP: Controlled attenuation parameter; LSM: Liver stiffness measurement 
and the difference was statistically significant $(\mathrm{p}<0.05)$; while fasting insulin, HOME- $\beta$ and $\Delta^{\mathrm{I} 30} / \Delta^{\mathrm{G} 30}$ increased after treatment than before treatment, and the difference was statistically significant $(\mathrm{p}<0.05)$, suggesting that blood sugar control and insulin secretion function are significantly better than before; body weight, BMI, waist circumference, waist-to-hip ratio, systolic blood pressure, diastolic blood pressure, TC, TG, HDL, LDL, ACR, blood uric acid, HOMA-IR, ALT, LSM and CAP before and after treatment were not significantly different $(\mathrm{p}>0.05)$, suggesting general physical indicators, blood pressure, blood lipids, serum uric acid, ACR and insulin resistance indicators did not improve significantly, while fatty liver related indicators such as ALT, LSM and CAP did not improve, as shown in TABLE 2.

After treatment, BMI, waist circumference, waist and hip of the dapagliflozin group decreased compared with before treatment, the difference was statistically significant $(p<0.05)$; the systolic blood pressure and diastolic blood pressure decreased compared with the previous, the difference was statistically significant $(\mathrm{p}<0.05)$, suggesting Blood pressure decreased after treatment; only triglycerides decreased in blood lipid levels, and the difference was significant $(\mathrm{p}<0.05)$; fasting blood glucose and $\mathrm{HbA1C}$ after treatment were lower than before treatment, the difference was statistically significant $(\mathrm{p}<0.05)$, while fasting insulin, HOME- $\beta$, and $\Delta^{130} / \Delta^{\mathrm{G} 30}$ after treatment increased compared with before treatment, the difference was statistically significant $(p<0.05)$, HOMA-IR decreased compared with before treatment, the difference was statistically significant $(\mathrm{p}<0.05)$, suggesting that after treatment Blood sugar control and insulin secretion function improved significantly, and insulin resistance improved. After treatment, ALT, LSM and CAP and other fatty liver related indicators were significantly lower than before treatment, and the difference was statistically significant $(p<0.05)$, suggesting fat after treatment The liver is improved, as shown in TABLE 2.

Compared with the control group, the dapagliflozin treatment group has statistical differences in $\Delta^{\text {Weight }}$, $\Delta^{\text {BMI }}, \Delta^{\text {Waist circumference }}, \Delta^{\text {Waist-hip ratio }}, \Delta^{\text {systolic blood pressure }}, \Delta^{\text {diastolic }}$ blood pressure $, \Delta^{\mathrm{TG}}, \Delta^{\mathrm{ACR}}, \Delta^{\text {serum uric acid }}$ and $\Delta^{\mathrm{HOME}-\mathrm{IR}}$ before and after treatment Academic significance $(\mathrm{p}<0.05)$. It is suggested that dapagliflozin gains more weight, BMI

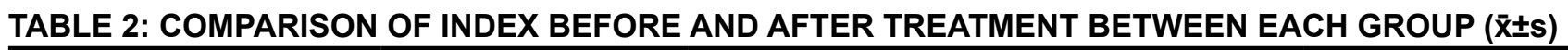

\begin{tabular}{|c|c|c|c|c|}
\hline \multirow[t]{2}{*}{ Item } & \multicolumn{2}{|c|}{ Control $(n=30)$} & \multicolumn{2}{|c|}{ Treatment $(n=30)$} \\
\hline & Before treatment & After treatment & Before treatment & After treatment \\
\hline $\operatorname{Sex}(M / F)$ & $23 / 7$ & & $24 / 6$ & \\
\hline Age (year) & $52.1 \pm 10.2$ & & $48.9 \pm 10.6$ & \\
\hline Weight (kg) & $76.45 \pm 9.17$ & $77.23 \pm 8.91$ & $78.51 \pm 12.65$ & $73.08 \pm 11.19$ \\
\hline BMI $\left(\mathrm{kg} / \mathrm{m}^{2}\right)$ & $25.82 \pm 6.67$ & $26.13 \pm 6.60$ & $27.71 \pm 3.29$ & $25.84 \pm 2.93^{*}$ \\
\hline Waist (cm) & $97.03 \pm 7.17$ & $96.27 \pm 6.71$ & $98.43 \pm 9.76$ & $91.16 \pm 9.18^{*}$ \\
\hline WHR & $0.96 \pm 0.06$ & $1.03 \pm 0.44$ & $0.97 \pm 0.07$ & $0.92 \pm 0.06^{*}$ \\
\hline SBP (mmHg) & $133.86 \pm 19.09$ & $133.80 \pm 17.71$ & $133.06 \pm 17.74$ & $124.8 \pm 12.97^{*}$ \\
\hline DBP (mmHg) & $88.6 \pm 9.59$ & $86.87 \pm 7.39$ & $90.13 \pm 11.40$ & $79.33 \pm 7.89^{*}$ \\
\hline TC (mmol/L) & $4.97 \pm 1.21$ & $4.88 \pm 1.07$ & $4.62 \pm 1.07$ & $4.36 \pm 0.88$ \\
\hline TG (mmol/L) & $3.52 \pm 0.87$ & $3.42 \pm 0.85$ & $3.72 \pm 3.23$ & $1.99 \pm 1.29^{*}$ \\
\hline HDL (mmol/L) & $3.30 \pm 1.11$ & $1.21 \pm 0.51$ & $1.95 \pm 5.23$ & $2.02 \pm 5.17$ \\
\hline LDL (mmol/L) & $3.31 \pm 2.02$ & $3.42 \pm 0.97$ & $2.98 \pm 0.69$ & $2.87 \pm 0.78$ \\
\hline ACR (mg/g) & $18.04 \pm 31.06$ & $19.78 \pm 34.85$ & $14.95 \pm 19.83$ & $10.39 \pm 2.49$ \\
\hline BUA (umol/L) & $334.1 \pm 78.46$ & $313.93 \pm 71.09$ & $356.90 \pm 83.15$ & $322.06 \pm 64.30$ \\
\hline ALT (mmol/L) & $38.14 \pm 39.67$ & $33.19 \pm 18.39$ & $36.5 \pm 19.80$ & $22.14 \pm 10.64^{*}$ \\
\hline FBG (mmol/L) & $11.24 \pm 3.57$ & $8.22 \pm 2.15^{*}$ & $13.05 \pm 3.68$ & $6.90 \pm 1.03^{*}$ \\
\hline Fins (uU/L) & $6.82 \pm 4.45$ & $8.064 \pm 3.45^{*}$ & $7.88 \pm 4.59$ & $9.00 \pm 4.81$ \\
\hline HOME-B & $19.63 \pm 16.13$ & $48.83 \pm 16.09^{*}$ & $19.50 \pm 13.50$ & $59.28 \pm 47.69^{*}$ \\
\hline HOME-IR & $3.98 \pm 2.21$ & $2.85 \pm 1.34$ & $4.41 \pm 2.50$ & $2.74 \pm 1.36^{*}$ \\
\hline$\Delta^{130} / \Delta^{\mathrm{G} 30}(\mathrm{uU} / \mathrm{mmol})$ & $1.03 \pm 1.63$ & $2.37 \pm 1.49^{*}$ & $1.73 \pm 2.40$ & $5.31 \pm 6.61^{*}$ \\
\hline HbA1C (\%) & $11.20 \pm 2.29$ & $7.06 \pm 1.72^{*}$ & $11.45 \pm 2.09$ & $6.92 \pm 0.58^{*}$ \\
\hline LSM (KPa) & $6.65 \pm 2.74$ & $6.71 \pm 2.87$ & $7.74 \pm 2.83$ & $6.03 \pm 2.52^{*}$ \\
\hline $\mathrm{CAP}(\mathrm{dB} / \mathrm{m})$ & $298 \pm 24.03$ & $264.76 \pm 32.74$ & $300.63 \pm 24.83$ & $254.40 \pm 31.3^{*}$ \\
\hline
\end{tabular}

Control: Treatment with Metformin; Treatment: treatment with dapagliflozin; BMl: Body mass index; WHR: Waist hip ratio; SBP: Systolic blood pressure; DBP: Diastolic blood pressure; TC: Total cholesterol; TG: Triglyceride; HDL-C: High-density lipoprotein cholesterol; LDL-C: Lowdensity lipoprotein cholesterol; ACR: Urinary albumin to creatinine ratio; BUA: Blood uric acid; FBG: Fasting plasma glucose; Fins: Fasting insulin; HOMA-B: B cell function index; HOMA-IR: Insulin resistance index; HbA1c: Glycosylated hemoglobin; ALT: Alanine aminotransferase; CAP: Controlled attenuation parameter; LSM: Liver stiffness measurement; Compared with before treatment, ${ }^{*} \mathrm{p}<0.05$ 
and waist circumference reduction than the control group, has a better effect on lowering blood pressure, TG and blood uric acid, and improves kidney disease and insulin resistance better than the control group. However, there was no statistical difference between $\Delta^{\mathrm{TC}}, \Delta^{\mathrm{HDL}}, \Delta^{\mathrm{LDL}}, \Delta^{\text {fasting blood glucose }}, \Delta^{\text {insulin }}, \Delta^{\text {HOME- } \beta}, \Delta^{\mathrm{I} 30} / \Delta^{\mathrm{G} 30}$ and $\mathrm{HbA} 1 \mathrm{C}$ between the two groups, suggesting that TC, HDL, LDL after dapagliflozin treatment There was no difference between the changes in blood lipid composition. However the metformin group was expected to decrease lipid levels as per the previous research data, contrary to this we have found insignificant changes in the levels of LDL and there was no significant difference between the two groups in terms of blood sugar control and improvement of pancreatic $\beta$-cell function, as shown in TABLE 3.

Compared with the control group, the $\Delta^{\mathrm{ALT}}, \Delta^{\mathrm{LSM}}$ and $\Delta^{\mathrm{CAP}}$ of the dapagliflozin group were significantly different before and after treatment, and the difference was statistically significant $(\mathrm{p}<0.05)$, suggesting that dapagliflozin treatment is beneficial to the improvement of non-alcoholic fatty liver in diabetic patients, as shown in TABLE 4.

\begin{tabular}{|c|c|c|}
\hline Item & Control $(n=30)$ & Treatment $(n=30)$ \\
\hline$\Delta$ Weight (kg) & $0.78 \pm 1.23$ & $-5.43 \pm 2.97^{\circ}$ \\
\hline$\triangle \mathrm{BMI}(\mathrm{kg} / \mathrm{m} 2)$ & $0.31 \pm 0 . .49$ & $-1.86 \pm 0.97^{*}$ \\
\hline$\Delta$ Waist $(\mathrm{cm})$ & $1.23 \pm 2.14$ & $-7.72 \pm 4.29^{*}$ \\
\hline$\triangle \mathrm{WHR}$ & $0.02 \pm 0.01$ & $-0.05 \pm 0.02^{*}$ \\
\hline$\triangle \mathrm{SBP}(\mathrm{mmHg})$ & $-0.07 \pm 3.95$ & $-8.27 \pm 10.75^{\star}$ \\
\hline$\triangle \mathrm{DBP}(\mathrm{mmHg})$ & $-1.77 \pm 4.62$ & $-10.8 \pm 9.25^{*}$ \\
\hline$\Delta \mathrm{TC}(\mathrm{mmol} / \mathrm{L})$ & $-0.90 \pm 0.25$ & $-0.26 \pm 1.36$ \\
\hline$\Delta \mathrm{TG}(\mathrm{mmol} / \mathrm{L})$ & $-0.086 \pm 0.16$ & $-1.72 \pm 2.93^{*}$ \\
\hline$\Delta \mathrm{HDL}(\mathrm{mmol} / \mathrm{L})$ & $-0.12 \pm 0.91$ & $0.07 \pm 0.59$ \\
\hline$\Delta \mathrm{LDL}(\mathrm{mmol} / \mathrm{L})$ & $0.11 \pm 0.32$ & $-0.10 \pm 1.00$ \\
\hline$\triangle \mathrm{ACR}(\mathrm{mg} / \mathrm{g})$ & $1.73 \pm 4,93$ & $-4.56 \pm 11.26^{*}$ \\
\hline$\triangle \mathrm{BUA}$ (umol/L) & $-1.67 \pm 12.25$ & $-34.83 \pm 40.75^{*}$ \\
\hline$\Delta \mathrm{FBG}(\mathrm{mmol} / \mathrm{L})$ & $-5.023 \pm 3.19$ & $-6.14 \pm 3.54$ \\
\hline$\Delta \mathrm{FIns}(\mathrm{uU} / \mathrm{L})$ & $2.45 \pm 3.01$ & $1.12 \pm 4.85$ \\
\hline$\triangle \mathrm{HOME}-\mathrm{B}$ & $36.20 \pm 14.69$ & $39.78 \pm 46.75$ \\
\hline$\triangle \mathrm{HOME}-\mathrm{IR}$ & $0.22 \pm 1.29$ & $-1.67 \pm 2.02^{*}$ \\
\hline$\Delta \mathrm{l} 30 / \Delta \mathrm{G} 30(\mathrm{uU} / \mathrm{mmol})$ & $1.34 \pm 1.34$ & $3.58 \pm 7.16$ \\
\hline$\Delta \mathrm{HbA1C}(\%)$ & $-4.14 \pm 2.30$ & $-4.54 \pm 2.17$ \\
\hline
\end{tabular}

Control: Treatment with Metformin; Treatment: treatment with dapagliflozin; $\Delta$ : Difference before and after treatment; BMI: Body mass index; WHR: Waist hip ratio; SBP: Systolic blood pressure; DBP: Diastolic blood pressure; TC: Total cholesterol; TG: Triglyceride; HDL-C: High-density lipoprotein cholesterol; LDL-C: Low-density lipoprotein cholesterol; ACR: Urinary albumin to creatinine ratio; BUA: Blood uric acid; FBG: Fasting plasma glucose; Fins: Fasting insulin; HOMA-B: $B$ cell function index; HOMA-IR: Insulin resistance index; HbA1c: Glycosylated hemoglobin; Compared with control group, ${ }^{*} \mathrm{p}<0.05$

Special Issue 7, 2020
TABLE 4: COMPARISON OF THE DIFFERENCE OF LIVER INDEXES BEFORE AND AFTER TREATMENT BETWEEN THE TWO GROUPS ( $\bar{x} \pm s)$

\begin{tabular}{lcc}
\hline Item & Control $(\mathbf{n}=30)$ & Treatment $(\mathbf{n}=30)$ \\
\hline$\triangle \mathrm{ALT}(\mathrm{U} / \mathrm{L})$ & $-4.95 \pm 27.09$ & $-14.35 \pm 16.25^{*}$ \\
$\triangle \mathrm{LSM}(\mathrm{KPa})$ & $0.06 \pm 0.25$ & $-1.70 \pm 0.36^{*}$ \\
$\Delta \mathrm{CAP}(\mathrm{dB} / \mathrm{m})$ & $-2.7 \pm 19.04$ & $-35.23 \pm 25.72^{*}$
\end{tabular}

Control: Treatment with Metformin; Treatment: treatment with dapagliflozin; $\Delta$ : Difference before and after treatment; ALT: Alanine aminotransferase; CAP: Controlled attenuation parameter; LSM: Liver stiffness measurement; Compared with control group, ${ }^{*} \mathrm{p}<0.05$

NAFLD is the most common comorbidity of type 2 diabetes. The liver B-ultrasound is usually used to diagnose fatty liver, but its monitoring results will be affected by the examiner's subjective judgment. Generally, liver steatosis can be detected by B-ultrasound when it exceeds $30 \%$. It is difficult to obtain quantitative indicators. Transient ultrasonic elastography technology is used to obtain the change of ultrasonic instantaneous elasticity for LSM and CAP is a non-invasive assessment of liver fibrosis and steatosis new method. A number of studies ${ }^{[9,10]}$ suggest that its sensitivity is higher than B-ultrasound and Computed Tomography (CT). Liver biopsy is considered to be the current gold standard for evaluating NAFLD ${ }^{[11]}$, and compared with liver biopsy, LSM and CAP have similar accuracy in diagnosing liver fibrosis and steatosis $^{[12,13]}$, which can be better evaluated. The degree of liver steatosis ${ }^{[14]}$. In this study, LSM, CAP, and alanine aminotransferase (ALT) were used as the main indicators for evaluating fatty liver. It is believed that these indicators can better reflect fatty liver disease and are economical, easy to obtain, and non-invasive.

Dapagliflozin is a new type of hypoglycemic drug and the first sodium-glucose cotransporter 2 (SGLT-2) inhibitor hypoglycemic drugs to be marketed in China. The drug has been proven to have a good hypoglycemic effect $^{[15]}$. Some studies also suggest that ${ }^{[16]}$ SGLT-2 inhibitors can reduce ALT levels in patients with type 2 diabetes and NAFLD, and may have the effect of treating NAFLD in patients with diabetes. There are not many studies on dapagliflozin in the treatment of diabetes with non-alcoholic liver steatosis, and there are fewer studies in China. This study found that the ALT, LSM, and CAP fatty liver-related indicators of patients with diabetes and non-alcoholic fatty liver disease after $12 \mathrm{w}$ of treatment with dapagliflozin were significantly better than before treatment, with statistically significant differences, while the control group had no related indicators before and after treatment. Compared with the control group, $\triangle \mathrm{ALT}, \triangle \mathrm{LSM}$, and $\triangle \mathrm{CAP}$ were significantly reduced before and after treatment, and 
the difference was statistically significant, indicating that dapagliflozin can reduce liver enzymes, liver fat content, and degree of liver fibrosis in patients with diabetes and NAFLD, Same results as Shimizu M et $a{ }^{[17]}$ and Aso $\mathrm{Y}^{[18]}$. Studies on animal models of non-alcoholic steatohepatitis ${ }^{[19,20]}$ show that SGLT-2 inhibitor drugs can improve fatty liver. Some clinical studies $^{[21,22]}$ also suggest that SGLT-2 inhibitors can improve non-alcoholic fatty liver disease in diabetic patients. These studies mainly involve two kinds of SGLT-2, dapagliflozin and canagliflozin, which are currently widely used in clinical practice. Inhibitor drugs and the use of NAFLD indirect indicators for evaluation, consistent with our research results. It suggests that dapagliflozin has the potential to treat non-alcoholic fatty liver in diabetic patients. Below we further explore the mechanism by which SGLT-2 inhibitors are beneficial to the improvement of nonalcoholic fatty liver in diabetic patients.

In this study, the fasting blood glucose and $\mathrm{HbA} 1 \mathrm{C}$ of the two groups of patients after treatment were significantly lower than before treatment, and HOME- $\beta$ and $\Delta \mathrm{I} 30 / \Delta \mathrm{G} 30$ were significantly higher than before treatment, and there was no significant difference between the two groups before and after treatment. . There were significant differences between the two groups before and after treatment of hepatic steatosis related indicators such as ALT, LSM and CAP. It is suggested that the decrease of blood sugar and the improvement of pancreatic $\beta$-cell function are not important factors for the improvement of fatty liver, which is consistent with the results of Eriksson JW et $a l .{ }^{[21]}$.

BMI, waist circumference and waist-hip in the dapagliflozin group were significantly lower than before treatment, but there was no significant difference in the control group before and after treatment, and the difference before and after treatment in the dapagliflozin group was significantly changed compared with the control group. It is suggested that dapagliflozin can significantly reduce the patient's BMI, waist circumference and waist-to-hip ratio, which is consistent with the results observed in the DECLAR study ${ }^{[23]}$. BMI, waist circumference and waist-to-hip ratio are closely related to the area of visceral fat ${ }^{[24]}$. In studies such as Tobita $\mathrm{H}^{[25]}$, dapagliflozin treatment can also reduce BMI, waist circumference and waist-to-hip ratio in patients with diabetes and NAFLD. Further studies have found that these changes are driven by body fat loss, and the body's skeletal muscle mass, moisture and the amount of protein does not decrease. After treatment with dapagliflozin, the body fat of diabetic patients decreases, especially the decrease of BMI, waist circumference and waist-to-hip ratio caused by the decrease of visceral fat, which reduces liver fat deposition and improves liver steatosis and fibrosis. It may be non-alcoholic fatty liver in diabetic patients. One of the important factors for improvement.

In the dapagliflozin treatment group in this study, triglyceride levels after treatment were significantly lower than before treatment, while other blood lipid components TC, LDL and HDL did not change before and after treatment. In the control group, all blood lipid components including TG did not change before and after treatment, which is consistent with the results of Jiang Lijuan et al. ${ }^{[26]}$. Animal experiments ${ }^{[27]}$ found that Sodium-glucose co-transporter-2 (SGLT2) inhibitor drugs reduce the expression levels of lipid synthesis genes adipocytes carboxylase $1(\mathrm{ACCl})$ and Sterol regulatory element-binding protein-1 cmRNA (SREBP$1 \mathrm{cmRNA}$ ), while increasing the expression levels of fatty acid oxidative decomposition genes Peroxisome Proliferator Activated Receptor Alpha (PPARa), Carnitine Palmitoyltransferase 1A (CPT-1A) and Microsomal triglyceride transfer protein mRNA (MTTP mRNA), thereby reducing liver lipid synthesis. Increase liver lipid utilization, fat deposition in the liver is due to the increase in the content of free fatty acids from food or fat cells, which increases the synthesis of lipids in the liver, while the oxidation of free fatty acids and the reduction of TG transfer are caused. Dapagliflozin may reduce liver lipid synthesis and increase liver lipid utilization, thereby improving liver steatosis in diabetic patients.

In this study, the dapagliflozin treatment group had a significant decrease in Insulin resistance index (HOME-IR) after treatment compared with before treatment, while the control group had no significant changes before and after treatment, suggesting that dapagliflozin can reduce insulin resistance in patients with diabetes and NAFLD, which is similar to Aso Y and others $^{[18]}$ The results of studies on dapagliflozin in the treatment of patients with type 2 diabetes and NAFLD are consistent. Dapagliflozin can increase insulin sensitivity and improve insulin resistance by lowering blood sugar, improving lipid metabolism, reducing weight and inflammation. There are also studies ${ }^{[28]}$ suggesting that SGLT2 inhibitors have anti-oxidative stress effects and help to improve insulin resistance. 
Insulin resistance is also an important factor in the occurrence and progression of NAFLD, suggesting that dapagliflozin can improve the liver steatosis of diabetic patients by improving insulin resistance, which may be one of the mechanisms.

To sum up, this study shows that dapagliflozin treatment is beneficial to the improvement of non-alcoholic fatty liver in patients with type 2 diabetes. The possible mechanism is related to dapagliflozin reducing BMI, waist circumference, waist-to-hip ratio, TG and insulin resistance, but specific The mechanism is still unclear, and it depends on further basic and clinical research to provide a clinical basis for the choice of hypoglycemic drugs for patients with diabetes and NAFLD.

\section{Acknowledgement:}

This work was supported by the Key Research and Development Plan Program of Anhui Province (No. 202004j07020050).

\section{Conflict of interests:}

The authors declared no conflict of interest.

\section{REFERENCES}

1. Williams CD, Stengel J, Asike MI, Torres DM, Shaw J, Contreras $\mathrm{M}$, et al. Prevalence of nonalcoholic fatty liver disease and nonalcoholic steatohepatitis among a largely middle-aged population utilizing ultrasound and liver biopsy: a prospective study. Gastroenterology 2011;140:124-31.

2. Zhu JZ, Zhou QY, Wang YM, Dai YN, Zhu J, Yu CH, et al. Prevalence of fatty liver disease and the economy in China: A systematic review. World J Gastroenterol 2015;21:5695-706.

3. Kwok R, Choi KC, Wong GL, Zhang Y, Chan HL, Luk AO, et al. Screening diabetic patients for non-alcoholic fatty liver disease with controlled attenuation parameter and liver stiffness measurements: a prospective cohort study. Gut 2016;65:1359-68.

4. Rhee EJ. Nonalcoholic Fatty Liver Disease and Diabetes: An Epidemiological Perspective. Endocrinol Metab 2019;34:22633.

5. Targher G, Lonardo A, Byrne CD. Nonalcoholic fatty liver disease and chronic vascular complications of diabetes mellitus. Nat Rev Endocrinol 2018;14:99-114.

6. Hazlehurst JM, Woods C, Marjot T, Cobbold JF, Tomlinson JW. Non-alcoholic fatty liver disease and diabetes. Metabolism 2016;65:1096-108.

7. Terami N, Ogawa D, Tachibana H, Hatanaka T, Wada J, Nakatsuka A, et al. Long-term treatment with the sodium glucose cotransporter 2 inhibitor, dapagliflozin, ameliorates glucose homeostasis and diabetic nephropathy in $\mathrm{db} / \mathrm{db}$ mice. PLos One 2014;9:e100777.

8. Chiba Y, Yamada T, Tsukita S, Takahashi K, Munakata Y, Shirai Y, et al. Dapagliflozin, a sodium-glucose co-transporter 2 inhibitor, acutely reduces energy expenditure in BAT via neural signals in mice. PLoS One 2016;11:e0150756.

9. Marengo A, Jouness RI, Bugianesi E. Progression and natural history of nonalcoholic fatty liver disease in adults. Clin Liver Dis 2016;20:313-24.

10. Tada T, Kumada T, Toyoda H, Mizuno K, Sone Y, Akita T, et al. Progression of liver fibrosis is associated with non-liverrelated mortality in patients with nonalcoholic fatty liver disease. Hepatol Commun 2017;1:899-910.

11. AlShaalan R, Aljiffry M, Al-Busafi S, Metrakos P, Hassanain M. Nonalcoholic fatty liver disease: Noninvasive methods of diagnosing hepatic steatosis. Saudi J Gastroenterol 2015;21:6470 .

12. Dezsofi A, Baumann U, Dhawan A, Durmaz O, Fischler B, Hadzic N, et al. Liver biopsy in children: position paper of the ESPGHAN Hepatology Committee. J Pediatr Gastroenterol Nutr 2015;60:408-20.

13. Kwok R, Tse YK, Wong GH, Ha Y, Lee AU, Ngu MC, et al. Systematic review with meta-analysis: non-invasive assessment of nonalcoholic fatty liver disease-the role of transient elastography and plasma cytokeratin-18fragments. Aliment Pharmacol Ther 2014;39:254-69.

14. De Ledinghen V, Vergniol J, Capdepont M, Chermak F, Hiriart $\mathrm{JB}$, Cassinotto $\mathrm{C}$, et al. Controlled attenuation parameter (CAP) for the diagnosis of steatosis: a prospective study of 5323 examinations. J Hepatol 2014;60:1026-31.

15. Mou LP, Jiang JJ, Zhang YP. Comparison of the efficacy and safety of dapagliflozin and linagliptin on overweight or obese type 2 diabetes patients with poorly controlled oral hypoglycemic drugs. Chin J Diabetes 2019;11:190-5.

16. Leiter LA, Forst T, Polidori D, Balis DA, Xie J, Sha S. Effect of canagliflozin on liver function test in patients with type 2 diabetes. Diabetes Metab 2016;42:25-32.

17. Shimizu M, Suzuki K, Kato K, Jojima T, Iijima T, Murohisa $\mathrm{T}$, et al. Evaluation of the effects of dapagliflozin, a sodiumglucose co-transporter-2 inhibitor, on hepatic steatosis and fibrosis using transient elastography in patients with type 2 diabetes and non-alcoholic fatty liver disease. Diabetes Obes Metab 2019;21:285-92.

18. Aso Y, Kato K, Sakurai S, Kishi H, Shimizu M, Jojima T, et al. Impact of dapagliflozin, an SGLT2 inhibitor, on serum levels of soluble dipeptidyl peptidase-4 in patients with type 2 diabetes and non-alcoholic fatty liver disease. Int J Clin Pract 2019;73:e13335.

19. Jojima T, Tomotsune T, Iijima T, Akimoto K, Suzuki K, Aso Y. Empagliflozin (an SGLT2 inhibitor), alone or in combination with linagliptin (a DPP-4 inhibitor), prevents steatohepatitis in a novel mouse model of nonalcoholic steatohepatitis and diabetes. Diabetol Metab Syndr 2016;8:1.

20. Komiya C, Tsuchiya K, Shiba K, Miyachi Y, Furuke S, Shimazu $\mathrm{N}$, et al. Ipragliflozin improves hepatic steatosis in obese mice and liver dysfunction in type 2 diabetic patients irrespective of body weight reduction. PloS one 2016;11:e0151511.

21. Eriksson JW, Lundkvist P, Jansson PA, Johansson L, Kvarnström M, Moris L, et al. Effects of dapagliflozin and n-3 carboxylic acids on non-alcoholic fatty liver disease in people with type 2 diabetes: a double-blind randomised placebocontrolled study. Diabetologia 2018;61:1923-34.

22. Bajaj HS, Brown RE, Bhullar L, Sohi N, Kalra S, Aronson R. SGLT2 inhibitors and incretin agents: Associations with alanine aminotransferase activity in type 2 diabetes. Diabetes Metab 2018;44:493-9.

23. Wiviott SD, Raz I, Bonaca MP, Mosenzon O, Kato ET, Cahn A, et al. The design and rationale for the Dapagliflozin Effect 
on Cardiovascular Events (DECLARE)-TIMI 58 Trial. Am Heart J 2018;6:83-9.

24. Wang HQ, Wang GY, Wang YB. Analysis of visceral fat area in patients with type 2 diabetes and visceral obesity. Chin Electron J Obes Metab Dis 2019;5:148-52.

25. Tobita H, Sato S, Miyake T, Ishihara S, Kinoshita Y. Effects of dapagliflozin on body composition and liver tests in patients with nonalcoholic steatohepatitis associated with type 2 diabetes mellitus: a prospective, open-label, uncontrolled study. Curr Ther Res 2017;87:13-9.

26. Jiang L, Liu F, Jiang Z, Li W, Lin P, Wang C, et al. Dapagliflozin improves lipid metabolism and visceral fat content in overweight and obese patients with type 2 diabetes. Journal of Shandong University (Medical Edition) 2019;57:87-93.

27. Honda Y, Imajo K, Kato T, Kessoku T, Ogawa Y, Tomeno $\mathrm{W}$, et al. The selective SGLT2 inhibitor ipragliflozin has a therapeutic effect on nonalcoholic steatohepatitis in mice. PLoS One 2016;11:e0146337.

28. Nakano S, Katsuno K, Isaji M, Nagasawa T, Buehrer B, Walker $S$, et al. Remogliflozin etabonate improves fatty liver disease in diet-induced obese male mice. J Clin Exp Hepatol 2015;5:190-8.

This is an open access article distributed under the terms of the Creative Commons Attribution-NonCommercial-ShareAlike 3.0 License, which allows others to remix, tweak, and build upon the work non-commercially, as long as the author is credited and the new creations are licensed under the identical terms

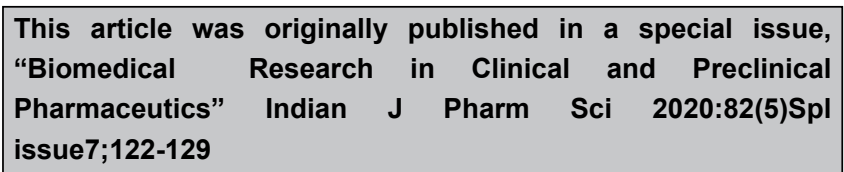

